\title{
Analysis of Viral Load Change in Case of HIV/AIDS Patients Under ART Follow-up in Arba Minch General Hospital
}

Markos Abiso Erango ( $\square$ markos.erango73@gmail.com )

Arba Minch University

Kabtamu Tolosie Gergiso

Arba Minch University

Sultan Hussen Hebo

Arba Minch University

\section{Research}

Keywords: Viral Load, Linear Mixed Effects Model, Longitudinal Data Analysis

Posted Date: March 1st, 2021

DOI: https://doi.org/10.21203/rs.3.rs-253684/v1

License: (c) (i) This work is licensed under a Creative Commons Attribution 4.0 International License.

Read Full License 


\title{
Analysis of Viral load change in Case of HIV/AIDS Patients under ART Follow-up in Arba Minch General Hospital
}

\author{
Markos Abiso Erango ${ }^{1}$, Kabtamu Tolosie Gergiso ${ }^{1}$ and Sultan Hussen Hebo ${ }^{2}$ \\ ${ }^{1}$ Department of Statistics, Arba Minch University, Arba Minch, Ethiopia \\ ${ }^{2}$ Department of Reproductive Health, Arba, Minch University, Arba Minch, Ethiopia
}

\begin{abstract}
Background: Monitoring human immunodeficiency virus plasma viral load over time is critical to identify virologic treatment failure in patients taking antiretroviral therapy. The aim of this study was to determine whether the overtime viral load changes depends on patient characteristic measured at baseline of human immunodeficiency virus patients at Arba Minch General Hospital.
\end{abstract}

Methods: This prospective follow up study was conducted using data obtained from medical records, patient interviews, and laboratory workup for six months. The study was employed among 152 adult patients that were selected by systematic random sampling. Longitudinal data analysis that accounts for the correlated nature of the data handled through linear mixed effect models were used to fit the data set in this study.

Result: The mean viral load declines over time for each of the adherence level groups. The estimates of linear $(p=0.0006)$ and quadratic visit time $(p=0.0256)$ effects and the baseline characteristics sex, age, adherence level, and Isoniazid preventive therapy had significant effects on change of viral load of patients over time.

Conclusion: In order to improve the status of the patient's viral load over time, considering the patients' differences in adherence to antiretroviral therapy, sex, age, and Isoniazid preventive therapy are important. 
Keywords: Viral Load, Linear Mixed Effects Model, Longitudinal Data Analysis

\section{Introduction}

Since the start of the epidemic of human immune deficiency virus (HIV), an estimated 77.3 million people infected and 35.4 million people died of acquired immune deficiency virus syndrome (AIDS) related illnesses. The vast majority of people living with HIV are located in low and middle income countries, with an estimated 66\% living in sub-Saharan Africa (SSA). Among this group 19.6 million are living in East and Southern Africa which saw 800,000 new HIV infections in 2017 [1]. In 2018, 23.3 million people with HIV (62\%) were accessing antiretroviral therapy (ART) globally, an increase of 1.6 million since 2017 and up from 8 million in 2010 [2].

Human immune deficiency virus treatment access is a key to the global effort to end AIDS as a public health threat. Currently, 12 million individuals are receiving antiretroviral therapy (ART), and a rapid scale up in the number of individuals receiving ART is in progress $(8,9)$. In an effort to scale up and decentralize ART services in Ethiopia, there are increasing numbers of health facilities providing ART and similarly the number of patients getting ART services is increasing (10).

The primary goals of initiating ART among HIV patients are to suppress HIV viral replication and to restore immune function. The clinical decision to check whether such goals have been achieved is made through periodic viral load testing and/or CD4 cell counting (11). Failure to achieve and maintain suppression can result in the development of drug resistance and also increases the risk of both horizontal and vertical viral transmission $(12$ - 14) 
In addition, a low viral load indicates that treatment is effective. A high viral load in a person on treatment indicates either that the medication is not being taken properly or that the virus is becoming resistant to the medication [3]. HIV patients with high viral loads are more infectious and lead to higher HIV transmission rates [4, 5].

Furthermore, plasma viral load monitoring is an important as a marker of response to ART, because a decline in viral load suggests that the patient is adherent to the regimen, that the appropriate doses are being administered, and that the virus is susceptible to the drugs in the regimen [7]. Viral load testing could increase in importance as a guide for clinical decisions on when to switch to second-line treatment and on how to optimize the duration of the first-line treatment regimen [8]. Recently published guidelines regarding the use of antiretroviral therapy now include the recommendation that both viral load and CD4 lymphocyte counts should be monitored regularly, and that plasma viral load should be reduced by as much and for as long as possible [8].

However, viral loads can fluctuate over time depending on patients' access to treatment, retention in care, response to HIV treatment, and medication adherence behavior [9]. In addition, cross-sectional viral load measures have invaluable for clinical and research purposes. Cross-sectional measures fail to capture cumulative plasma HIV burden longitudinally. Thus, longitudinal data analysis is recommended for cumulative viral load measures. Therefore, the aim this study was to determine whether the overtime viral load changes depends on patient characteristic measured at baseline of human immunodeficiency virus patients at Arba Minch General Hospital. 


\section{Materials and Methods}

\section{Study area and design}

The study was conducted in Arba Minch General Hospital, Arba Minch town, Gamo zone, Southern Ethiopia. A facility based prospective follow up study was conducted on HIV-infected adult patients receiving ART at the Arba Minch general hospital from March 1, 2017 to Feb. 30, 2018.

\section{Source and study populations}

Source population was all adult people living with HIV who were enrolled on first line ART regimen at Arba Minch General Hospital from March 01, 2017 to February 30, 2018. The study population was all adult living with HIV who was enrolled on first ART regimen at Arba Minch General Hospital and who fulfill the inclusion criteria. The study included patients with documented HIV infection, initiation of ARV drugs for the first time after March 1, 2017 and age $\geq 15$ years. But, patients with previous history of exposure for antiretroviral treatment were excluded from the study.

\section{Sample size determination and sampling technique}

The calculation of the required sample size for the number of patients needed for viral load testing in this study is calculated by using free and open source statistical software for epidemiological statistics called Open-Epi based on the following assumptions. Based on the study done in public HIV treatment settings of Haiphong, Vietnam, among those who have viral load suppression, the proportion of patients with poor adherence during ART was $62.3 \%$ and good adherence during ART was 37.7\% (14). In addition, 95\% Confidence interval, $80 \%$ power and a 
minimum detectable alternative of $\pm 5 \%$. Under these circumstances, the calculated sample size was 146 participants. With an assumed non-response rate of $4 \%$, the sample size needed for this study was $146 \mathrm{X}$ $0.04+146=152$ participants.

In this study, systematic sampling technique with $\mathrm{k}^{\text {th }}$ interval (i.e. $\mathrm{k}=\mathrm{N} / \mathrm{n}$ ), $\mathrm{k}=263 / 152=1.73 \approx 2$ (i.e. every other patients were selected). The first study participant was selected randomly from the first patients who visited the clinic.

\section{Study variables}

The response variable considered for this study was the viral load. It was measured approximately every 1-month interval. The viral load of patient was recorded seven times at months $0,1,2,3,4,5$, and 6 . The covariate variables that are assumed to influence the longitudinal response of the patient included in the model are: Sex, Age, Residence, Marital Status, Religion, BMI, visit time, Employment status, Educational Status, adherence to ART treatment, Initial regimen prescribed, Substance use, Disclosure status, Baseline CD4 count, WHO clinical staging prior to ART, Other opportunistic infections, functional status, Cotrimoxazole prophylaxis, and Isoniazid prophylaxis.

\section{Data collection procedures}

After written consent obtained, using data collection tools, the data were collected on sociodemographic, baseline clinical and treatment related characteristics of the participants by their clinical attendant (trained data collector) starting from the commencement of the care. The patient's information was obtained from the participants, their data base and medical charts. Additional information was collected during the 6 months follow up of the study participants. 
Specimen for the laboratory tests of CD4 and viral load tests was collected by the trained laboratory staff at the facility. For both tests $4-5 \mathrm{ml}$ of whole blood was drawn from each participant using vacutainer tube separately with anticoagulant following standard venipuncture protocols for viral load testing. Plasma sample was assayed for the presence of HIV RNA using Amplicor Monitor standard assay, version 1.5 (Roche Molecular Systems).

\section{Data processing and analysis}

The statistical software used in this study was the Statistical Analysis Software (SAS) version 9.4. Data was cleaned before subjected to analysis. The exploratory data analysis was conducted in order to acquire knowledge about the mean and variance structures of the outcome variable viral load count over time, the summary statistics of the viral load count on different occasions were tabulated. In order to get an idea about the correlation structure to describe how measurements within a subject correlate, the correlation matrix of response in different occasions was also tabulated. The graphical presentation techniques such as individual profiles plot and mean profile plot were drawn in order to have an idea about the evolution of viral load count over time. Also, linear mixed model was used under this study.

\section{Linear Mixed-Effects Model}

The continuous outcome variable viral load count contains measured at months $0,1,2,3$, 4, 5, and 6. Since measurements were taken from the same subject over time, observations cannot be considered as independent. So, appropriate random effect models that account for the correlated nature of the data was used. The random-effects approach is extending 
the univariate linear regression model to longitudinal settings based on subject-specific regression model [11].

A longitudinal model is the estimation of changes in response over time and testing whether these changes are covariate dependent [12]. Special methods of statistical analysis are needed for longitudinal data because the set of measurements on one patient tend to be correlated, measurements on the same patient close in time tend to be more highly correlated than measurements far apart in time, and the variability of longitudinal data often changes with time. These potential patterns of correlation and variation may combine to produce a complicated covariance structure. This covariance structure must be taken into account to draw valid statistical inferences.

The general form of the linear mixed model employed for which assumes that the outcome vector $\boldsymbol{Y}_{i}$ of all $n_{i}$ outcomes for subject $i$ satisfies [13]

$$
\boldsymbol{Y}_{i}=\boldsymbol{X}_{i} \boldsymbol{\beta}+\boldsymbol{Z}_{i} \boldsymbol{b}_{i}+\epsilon_{i}
$$

in which $\boldsymbol{\beta}$ is a vector of population average regression coefficients, called fixed effects, and where $\boldsymbol{b}_{i}$ is a vector of subject-specific regression coefficients. The $\boldsymbol{b} \boldsymbol{i}$ are assumed normal with mean vector $\mathbf{0}$ and covariance $D$, and they describe how the evolution of the $i^{\text {th }}$ subject deviates from the average evolution in the population. The matrices $\boldsymbol{X}_{i}$ and $\boldsymbol{Z}_{i}$ are $\left(n_{i} \times \mathrm{p}\right)$ and $\left(n_{i} \times \mathrm{q}\right)$ matrices of known covariates. Note that $\mathrm{p}$ and $\mathrm{q}$ are the numbers of fixed and subject-specific regression parameters in the model, respectively. The residual components $\epsilon_{i}$ are assumed to be independent $N\left(\mathbf{0} ; \Sigma_{i}\right)$, where $\Sigma_{i}$ depends on $i$ only through its dimension $n_{i} . \mathrm{D}$ and $\Sigma_{i}$ are the variance components, where $D$ is the covariance matrix of random effects, and $\Sigma_{i}$ is the 
measurement error matrix. The vector $\boldsymbol{\beta}$ and $\boldsymbol{b}_{i}$ are the fixed effects (the predicted variables are supposed to have the same effects for all individuals) and the random effects (the predicted variables also have an additional individual-specific effect, allowing variation between individuals), respectively.

To assess the need for serial correlation inclusion, the criterion of fitting linear mixed models with the same mean and random-effects structure was used as proposed by Verbeke and Molenberghs [11].

\section{Model Selection Criteria}

A key part of the analysis of data is model selection, which often aims to choose a parsimonious model. To have an appropriate model for the linear mixed model most commonly known model selection criterions; Akaike Information Criterion (AIC) and the Bayesian Information Criterion (BIC) [14] were considered for this study.

\section{Ethical considerations}

The letter of ethical clearance was obtained from the institutional review board (IRB) of College of Medicine and Health Sciences in Arba Minch University. Written consent was obtained from all study participants for blood draws and interviews. Participants with a high likelihood of virologic failure prioritized for tailored individualized treatment preparation and other interventions to improve treatment outcomes in the hospital. 


\section{Results}

\section{Exploratory data analysis}

The baseline socio-demographic, clinical and chemo prophylaxis characteristics of adult enrolled on first line ART were presented in Table 1 below. Out of total 152 HIV patients, 89 (58.55\%) were male and 63(41.45\%) were female. Most of the study participant 82(53.65\%) were married. The majority of participants was where lived at urban 114(75\%), and employed 100(65.79\%).Sixty-seven (44.08\%) of patients experienced good adherence level to treatment. In addition, $94(61.84 \%)$ of them had history of substance use at initiation of ART and 102 (67.11\%) disclosed their HIV status to their family members. At baseline, $55(36.18 \%)$ of the study participants were categorized in stage one of WHO staging category and 23(15.13\%) were ambulatory. Only 9(5.92\%) patients were received ABC/3TC/NVP or EFV initial regimen. Fourth three (28.29\%) had experienced CPT therapy.

The summary statistics at baseline variables and viral load over time were presented in Table 2. Regarding the adherence level composition of patients, the mean age whose patients had experienced good, fair, and poor adherence level are $31.85, \quad 31.42$ and 34.04 respectively. The mean CD4 of patients with poor adherence level to treatment was 158.8 . this shows that the mean CD4 of patients with poor adherence level is smaller as compared to good and fair adherence level groups. Moreover, the mean viral load declines over time for each of the adherence level groups. The patients with the good adherence level have lowest viral load with mean 681.82 for the last visit. 
Table 2: Summary measures of covariates and response at each time points of adult newly enrolled in ART at Arba Minch General Hospital, Gamo zone, Southern Ethiopia, 2018

The next step in longitudinal studies address the relationship of a response with explanatory variables, often including time. The individual profiles, the average evolution, the variance function, and the correlation structure are very helpful tool in the selection of appropriate models. In the following sections exploratory analysis for the data sets considered.

\section{Individual Profile}

Figure 1, shows how the viral load of HIV/AIDS patient's evolves overtime. The evolution of viral load count for each individual suggests variability between subjects. This plot also shows that different baseline values at the start of the study were recorded which indicated that a random intercept in the model. Additionally, the viral load count evolution seems different across subjects and this again suggests the requirement of a random slope during mixed model fitting. See Figure 1 below

Figure 1: Individual profiles of Viral Load at 7 visit time points

\section{Mean Structure}

The average values are computed at each point separately and connected. This will give idea as to how the profile for a number of the population evolves over time. In order to see the average evolution of the viral load over time and to have some idea what the mean structure looks like, a plot of the average evolution overtime was depicted. The average evolution 
describes, how the profile of a number of relevant subpopulations (or the population as a whole) evolves over time. From Figure 2 (a), the results of this exploration will be useful in order to choose a fixed-effects structure of the linear mixed model. The average of the profiles strictly decreasing from baseline study to month 2 and then seems to slightly decrease from month 2 to month 6. From individual profiles and the average evolution plots, our results suggest modeling the viral load as quadratic function overtime. This results in an average intercept and an average linear as well as quadratic time effect. Moreover, the average profile seems to indicate that a difference in viral load exists between sex, adherence

level groups Figure 2 (c \& d) and disclosure status, functional status, history of substance use, CTP therapy and initial regimen groups Figure 3 during a visit time.

\section{The Variance Structure}

Besides the average evolution, the evolution of variance over time is important to build an appropriate subject-specific or marginal longitudinal model. The plot of the average evolution of variance as a function of time was done to identify the variance structure Figure 2 (b). The overall variance function seems relatively stable, showing a decreasing pattern over time and hence a constant variance model could be a plausible starting point for longitudinal modeling approach.

Figure 2: Overall average evolution (a), over all variance evolution (b), mean evolution by sex (c) and mean evolution by adherence level (d) for viral load during a visit time. 
Figure 3: Average evolution of viral load, mean evolution by disclosure status (a), mean evolution by functional status (b), mean evolution by history of substance use (c), mean evolution by CTP therapy (d) and mean evolution by initial regimen (e).

\section{The Correlation Structure}

The correlation structure describes how measurements within a subject correlate. The correlation matrix presented in Table 3 below shows the pairwise correlation between measurements at any pair of time points. It was observed that there were positive correlations between the pair of measurements. Also, scatter plot matrices are a way to roughly determine if we have a linear correlation between multiple variables. The scatter plot matrix displayed in Figure 4 below, shows that viral load count at months $(0,1,2,3,4,5$, and 6$)$ are strongly correlated. So, we could consider the correlation of data in the model.

Figure 4: Scatter plot matrix for the Viral Load at 7 visit time points

\section{Result of Linear Mixed Model}

The outcome variable of interest is the viral load by which evolution over time may be assessed. Based on results of exploratory data analysis, the evolution of the viral load was assumed to have a linear and quadratic function over time effect as a preliminary mean structure and unstructured variance-covariance structures seemed a plausible starting for performing linear mixed model. The models were compared using the likelihood ratio test based on REML and results showed that there was no need of including the serial correlation.

The possibility of random-effects structure reduction was also assessed using the likelihood ratio test (mixture of chi-squares with equal weights 0.5 ) by deleting random effects in a 
hierarchical way starting from the highest order time effect comparing it with the previous model. After this, simultaneous contrast statements were used to explore the possibility of reducing the mean structure, starting from the unstructured mean effect which was rejected. In all cases, the possibility of simplification of random effects from the models was the only model with random intercept. The fixed effects considered were sex, age, residence, marital status, BMI, employment status, educational Status, adherence to ART treatment, initial regimen prescribed, substance use, disclosure status, CD4 count, WHO clinical staging prior to ART, other opportunistic infections, functional status, CPT therapy, and INH therapy, the linear, and the quadratic time effect of viral load, and the interaction sex, adherence level, disclosure status, history of substance use, CTP therapy with linear time effects. After model selection, the final model in Table 4 with AIC= 19517.9 obtained:

From the fitted result of linear mixed model of Table 4 below, it can be seen that the estimates of linear and quadratic visit time effects and the baseline characteristics sex, age, adherence level, and INH therapy effects had significant effect on viral load measurements. However, no significant difference between marital status, who stage, education level, disclosure status, history substance use, functional status at start ART, initial regimen, and CTP therapy groups. Also, BMI and CD4 had no significant effect on the viral load of HIV/AIDS patients.

The fitted result of the linear mixed model at baseline the viral load of the female group was 9276.09 greater than male patients. Baseline the viral load of fair adherence level group was 1411.60 greater and poor adherence level patients group was 21621 greater viral load measurements in comparison with good adherence level used group. Furthermore, the model showed with a one-year increase in age of the patients decreases the mean viral load 
measurements by 802.15 and linear time also has negative effects on the mean change of viral load measurements.

\section{Discussion}

This study was designed to fit a model of how adherence level influence viral load over visit time and whether the evolution of viral load depends on patient characteristic measured at baseline of HIV/AIDS patients at Arba Minch General Hospital.

This study showed that variable sex had significant effect on viral load. The viral load of the female group was greater than male patients. A related study by Adal et al.[15] also showed an association between sex and HIV RNA load. In another study by Hewitt et al.[16] it was found that women appear to have an increased risk of progression to AIDS compared with men with the same viral load. However, study aimed to assesses the use of both viral load (HIV RNA) and CD4 cell count in the monitoring of HIV/AIDS progression, it was found that there was no sex effect on the progression of HIV based on viral load levels [6]. Moreover, the present study identified that the baseline age and INH therapy were significantly associated with the change in viral load over time.

In previous a study, it was investigated that the level of adherence to HAART is closely associated with suppression of the HIV viral load in plasma [17]. In the current study also found that adherence level had significant effect on viral load. Baseline the viral load of fair and poor adherence level group were greater viral load measurements in comparison with good adherence level used group. 
In conclusion, the viral load of HIV/AIDS patients is significantly determined by the visit time, sex, age, adherence level, and INH therapy effects of patients. In order to improve the patients viral load over time, considering the difference of patients' sex, age, adherence to ART and INH therapy is important.

\section{Declarations}

\section{Authors' contributions}

KT, SH and MA conceived and designed the study, developed data collection instruments and supervised data collection. They participated in the testing and finalization of the data collection instruments and coordinated the study progress. KT, SH and MA performed the statistical analysis and wrote all versions of the manuscript. All authors read and approved the final manuscript.

\section{Acknowledgments}

We would like to acknowledge Arba Minch General Hospital administrator and data collectors who contributed to this work. We would also like to extend our gratitude to Arba Minch University for all support and opportunity provided for us to conduct this study.

\section{Competing interests}

The authors declare that they have no competing interest Availability of data and materials The raw data documents are available upon request from the corresponding author.

\section{Consent to publish}

Not applicable. 


\section{Funding}

Not applicable

\section{References}

1. Global HIV and AIDS statistics. (2017). https://www.avert.org/global-hiv-and$\underline{\text { aidsstatistics }}$

2. The Global HIV/AIDS Epidemic. (2019). https://www.hiv.gov/hivbasics/overview/data$\underline{\text { and-trends/global-statistics }}$

3. Joint United Nations Programme on HIV/AIDS (UNAIDS). (2016). The need for routine viral load testing. https://www.unaids.org/sites/default/files/media asset/JC2845 en.pdf

4. HIV \& AIDS. (2017). Viral Load. https://www.aidsmap.com/about-hiv/viral-load

5. Blaser, N., Wettstein, C., Estill, J., Vizcaya, L. S., Wandeler, G., Egger, M., \& Keiser, O. (2014). Impact of viral load and the duration of primary infection on HIV transmission: systematic review and meta-analysis. AIDS (London, England), 28(7), 1021.

6. Shoko, C., \& Chikobvu, D. (2019). A superiority of viral load over CD4 cell count when predicting mortality in HIV patients on therapy. BMC infectious diseases, 19(1), 169.

7. AIDSinfo. (2019). Guidelines for the Use of Antiretroviral Agents in Pediatric HIV Infection. https://aidsinfo.nih.gov/contentfiles/lvguidelines/glchunk/glchunk 59.pdf

8. Calmy, A., Ford, N., Hirschel, B., Reynolds, S. J., Lynen, L., Goemaere, E., ... \& Rodriguez, W. (2007). HIV viral load monitoring in resource-limited regions: optional or necessary?. Clinical infectious diseases, 44(1), 128-134.

9. Crepaz, N., Tang, T., Marks, G., \& Hall, H. I. (2017). Changes in viral suppression status among US HIV-infected patients receiving care. Aids, 31(17), 2421-2425. 
10. Huong, D. T. M., Bannister, W., Phong, P. T., Kirk, O., \& Peters, L. (2011). Factors associated with HIV-1 virological failure in an outpatient clinic for HIV-infected people in Haiphong, Vietnam. International journal of STD \& AIDS, 22(11), 659-664.

11. Verbeke, G., \& Molenberghs, G. (2000). Linear Mixed Models for Longitudinal Data, Springer, New York.

12. Molenberghs, G. and Verbeke, G. (2005). Models for Discrete Longitudinal Data, Springer Series in Statistics, Springer New York.

13. Laird, N. M. \& Ware, J. H. (1982). Random effects models for longitudinal data. Biometrics 38, 963\{974

14. Weiss, R. E. (2005). Modeling longitudinal data. Springer Science and Business Media.

15. Adal, M., Howe, R., Kassa, D., Aseffa, A., \& Petros, B. (2018). Associations of gender and serum total cholesterol with CD4+ $\mathrm{T}$ cell count and HIV RNA load in antiretroviralna"ive individuals in Addis Ababa. BMC public health, 18(1), 943.

16. Hewitt, R. G., Parsa, N., \& Gugino, L. (2001). The role of gender in HIV progression. AIDS READER-NEW YORK-, 11(1), 29-33.

17. Bangsberg, D. R., Hecht, F. M., Charlebois, E. D., Zolopa, A. R., Holodniy, M., Sheiner, L., ... \& Moss, A. (2000). Adherence to protease inhibitors, HIV-1 viral load, and development of drug resistance in an indigent population. Aids, 14(4), 357-366 
Figures

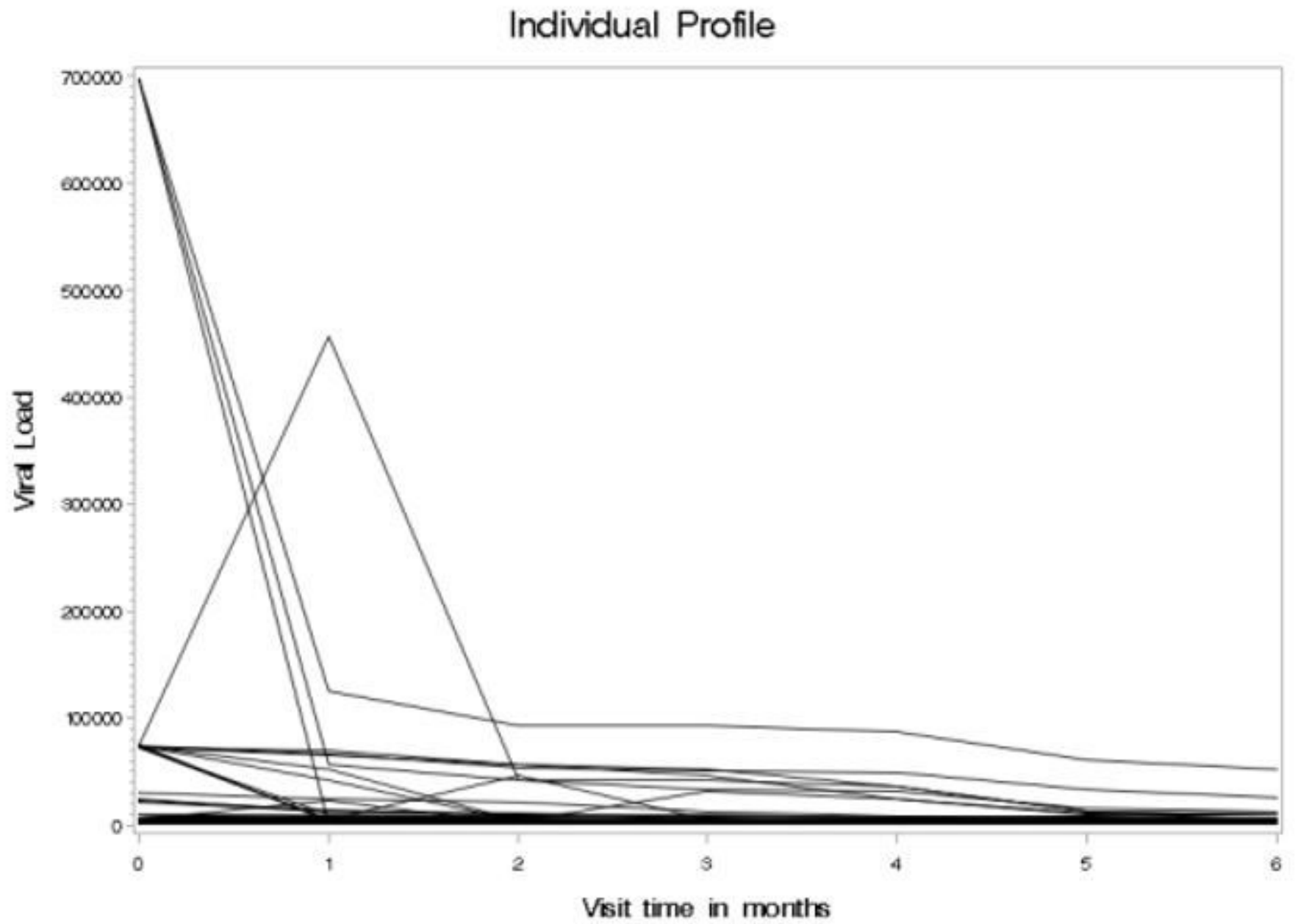

Figure 1

Individual profiles of Viral Load at 7 visit time points 


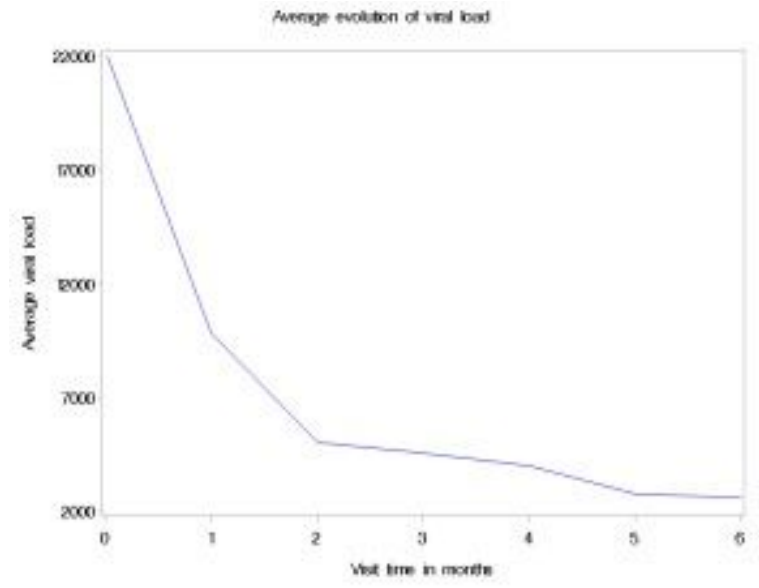

(a)

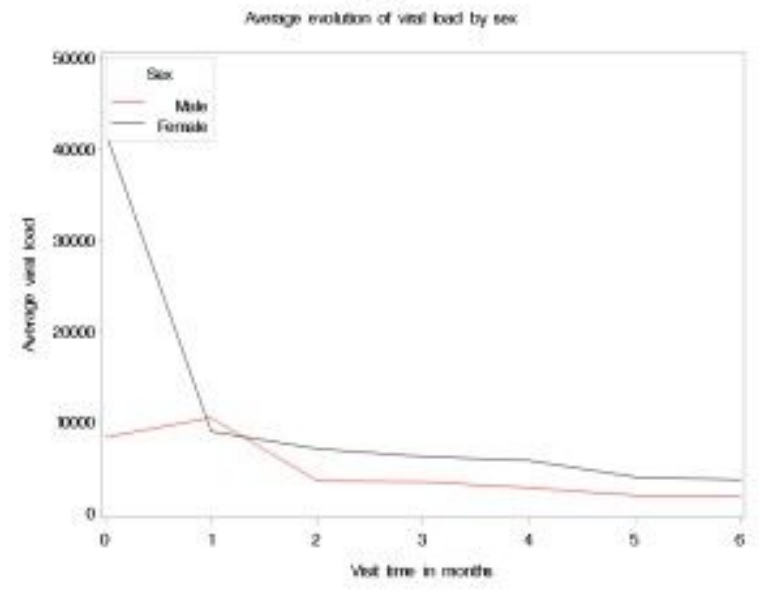

(c)

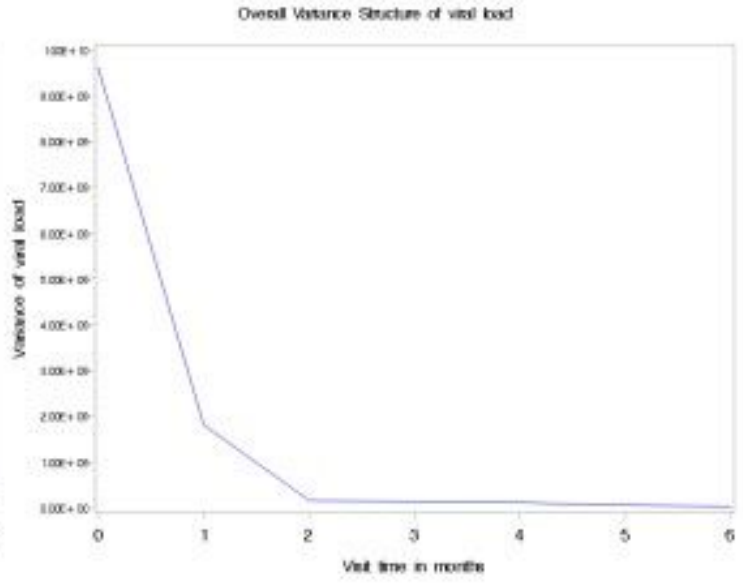

(b)

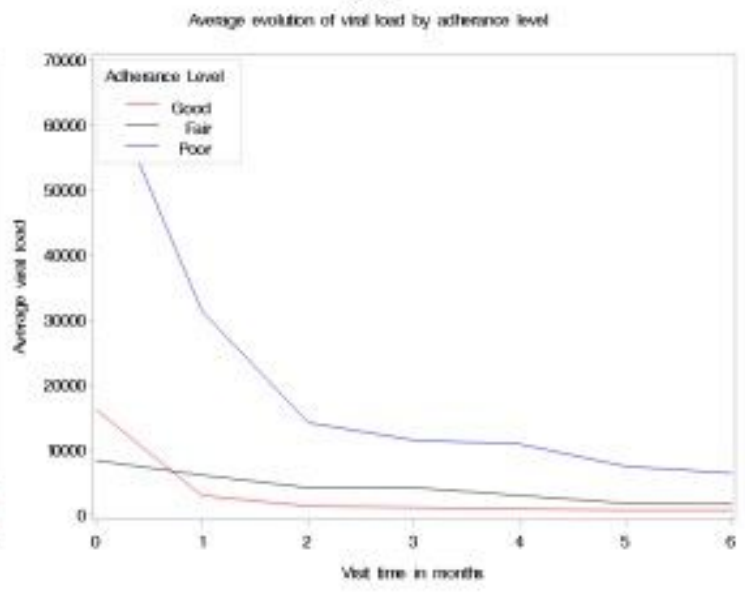

(d)

Figure 2

Overall average evolution (a), over all variance evolution (b), mean evolution by sex (c) and mean evolution by adherence level (d) for viral load during a visit time. 


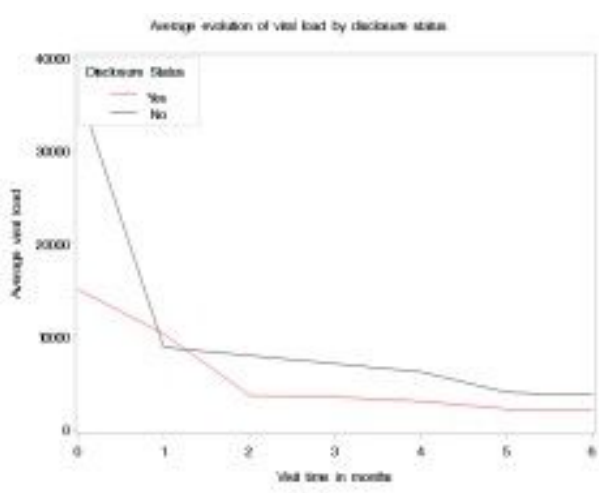

(a)

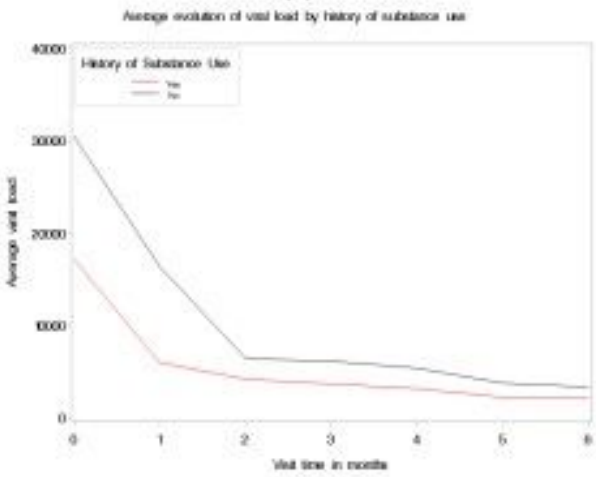

(c)

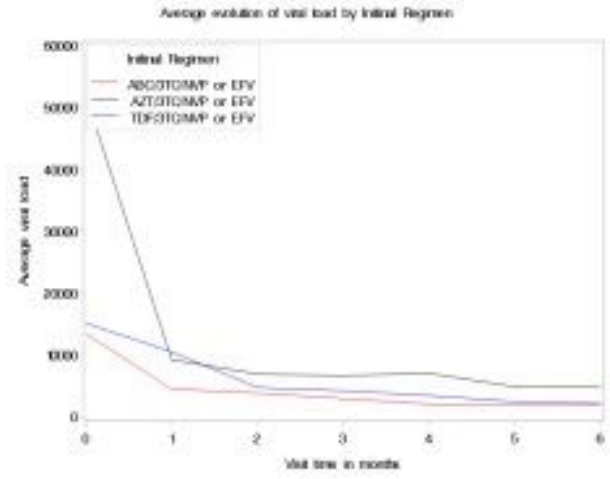

(e)

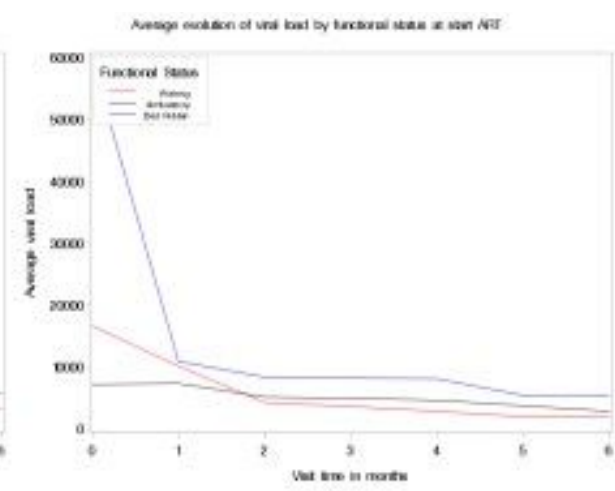

(b)

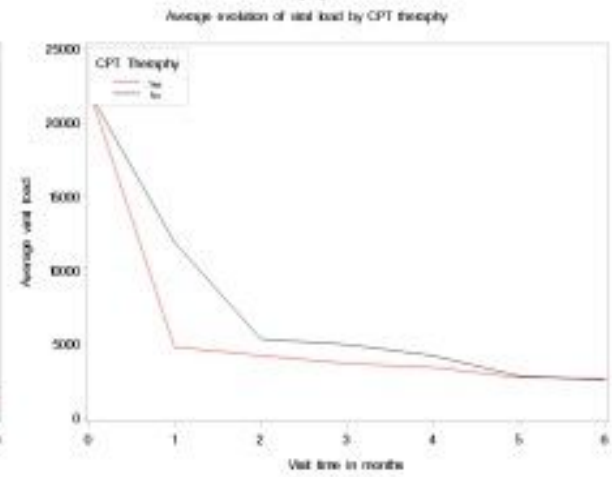

(d)

\section{Figure 3}

Average evolution of viral load, mean evolution by disclosure status (a), mean evolution by functional status (b), mean evolution by history of substance use (c), mean evolution by CTP therapy (d) and mean evolution by initial regimen (e). 


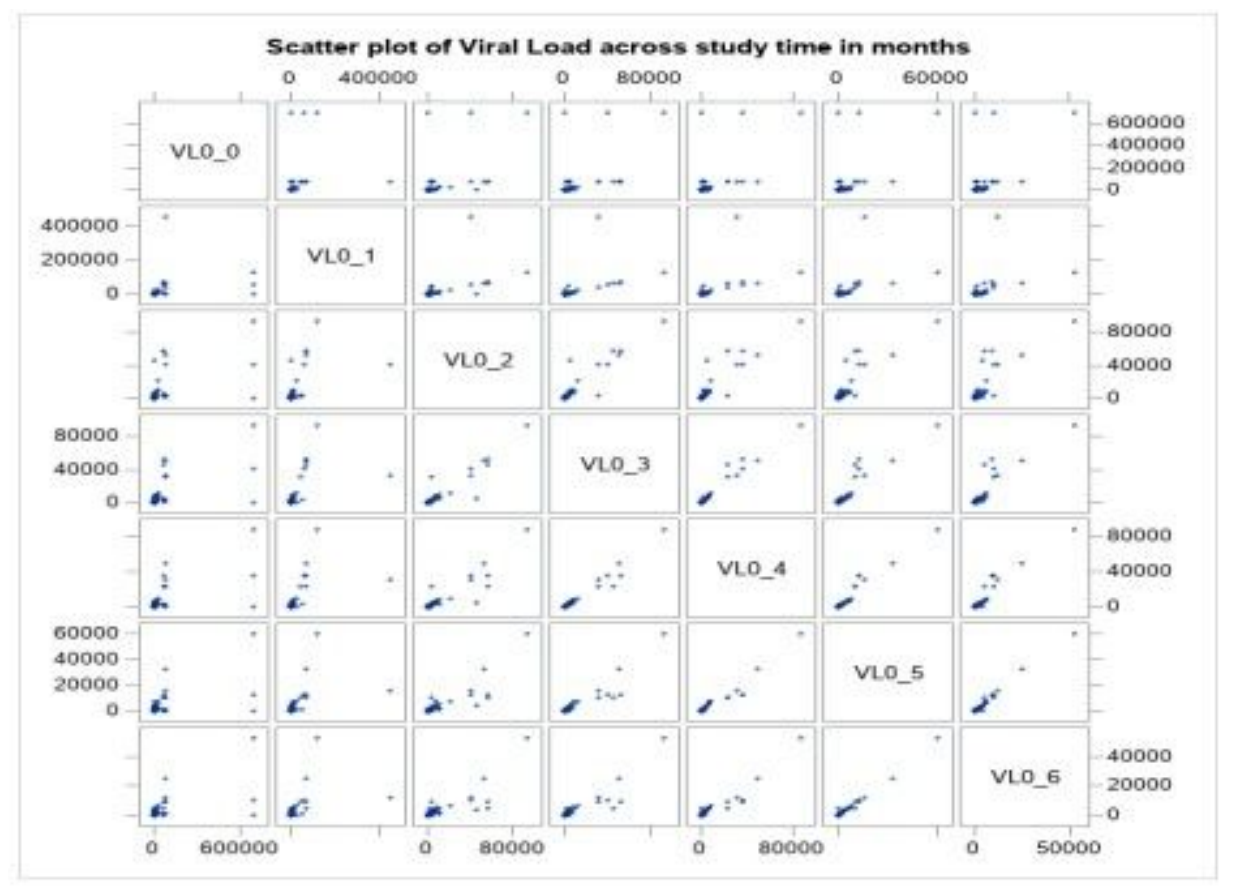

Figure 4

Scatter plot matrix for the Viral Load at 7 visit time points

\section{Supplementary Files}

This is a list of supplementary files associated with this preprint. Click to download.

- Table4.docx 